\title{
Orientaciones y retos en la formación docente para la transformación educativa
}

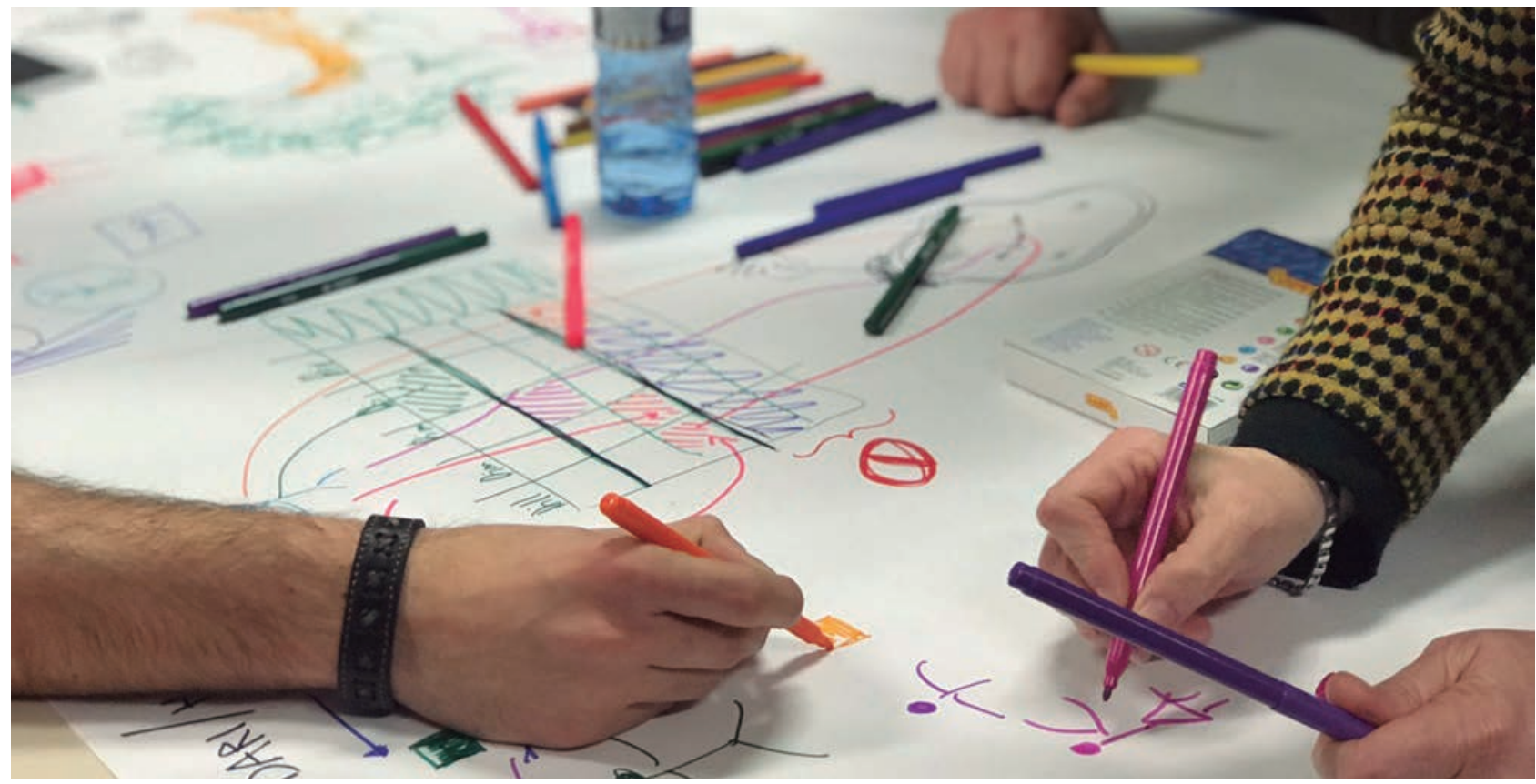

La transformación y la innovación educativa ha de estar guiada por el propósito de nuestra propuesta: acompañar el desarrollo integral de nuestro alumnado para que crezcan como ciudadanos y ciudadanas globales comprometidas con la reconciliación, la justicia y el cuidado de la casa común. Para responder a dicho propósito es necesario repensar la escuela, cambiar las prácticas educativas y mejorar los procesos de enseñanza y aprendizaje, lo que requiere a su vez la actualización de las experiencias formativas de nuestros educadores para que potencien su desarrollo profesional y transformen su identidad docente. Desde la experiencia en nuestro proyecto de transformación educativa en Jesuïtes Educació,

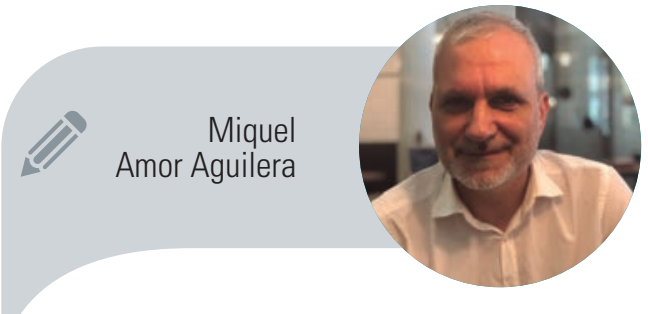

Director de formación e innovación miquel.amor@fje.edu https://www.fje.edu/edu

$\boldsymbol{\theta}$

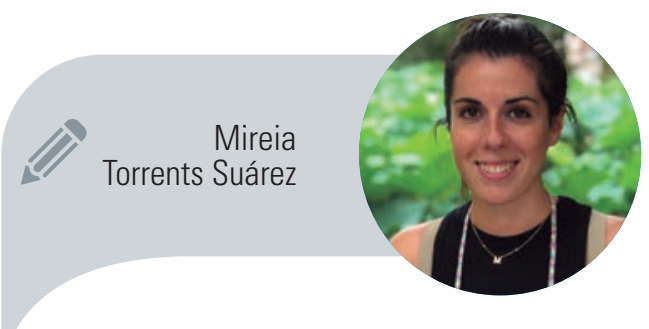

Responsable de proyectos de innovación educativa mireia.torrents@fje.edu https://www.fje.edu/edu $\boldsymbol{\theta}$ compartimos la importancia de acercar espacios de reflexión sobre la propia práctica en entornos de colaboración entre docentes. 

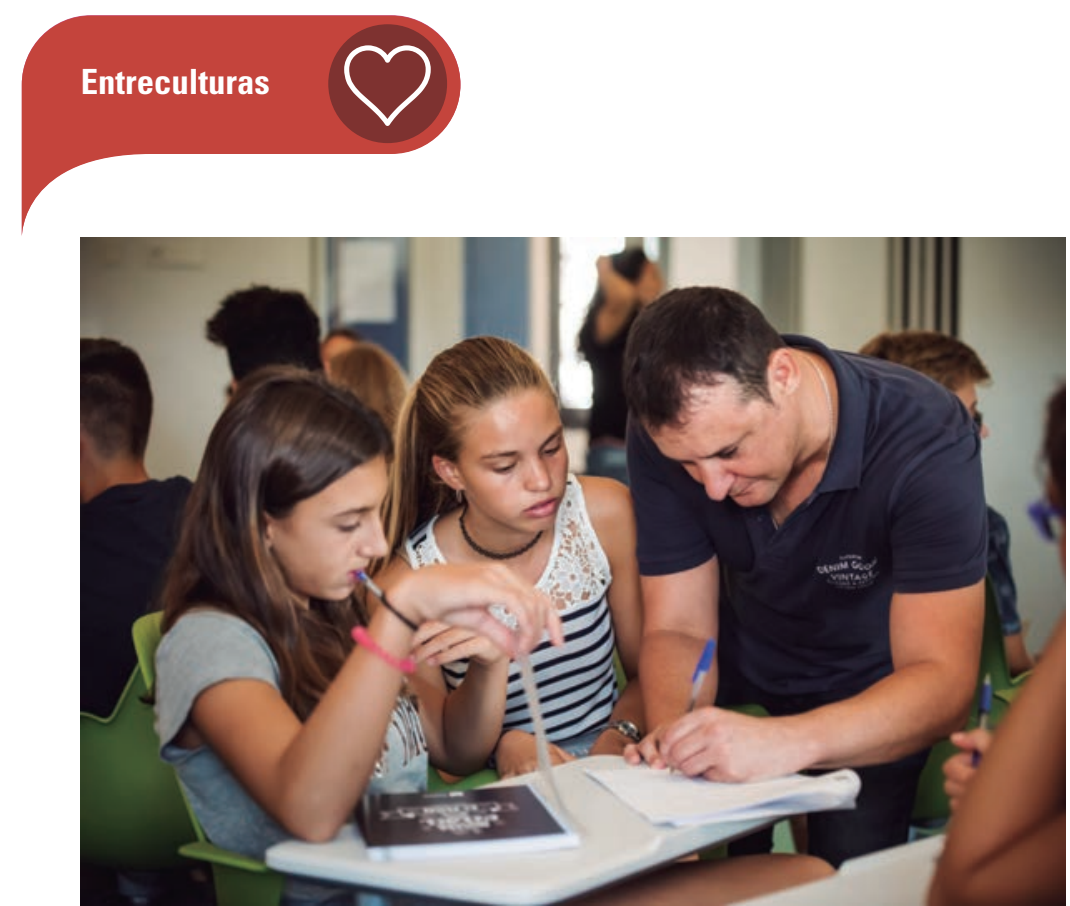

Docente de Jesuites Lleida con alumnas de TQE (tercero y cuarto de secundaria)
La misión que se nos encomienda como escuelas pertenecientes a la Compañía de Jesús es educar personas que puedan crecer y vivir con plenitud en este mundo y tengan la capacidad y el deseo de transformarlo a favor del bien común.

Desde este posicionamiento, la educación no se sitúa tan solo en la mera excelencia académica y la adquisición de conocimientos. Acompañar a los y las jóvenes en la creación de un futuro esperanzador pasa por el desarrollo también de competencias, valores, actitudes y habilidades sociales que les conduzcan a una mayor conciencia y compromiso en la transformación social. Eso implica reconocer también un papel relevante a la educación en las diferentes problemáticas, locales y globales, de los ámbitos social, político, cultural, económico y ambiental.

Siendo fieles a la cultura innovadora propia de la Compañía de Jesús, las 8 escuelas jesuitas de Cataluña que conforman la fundación Jesuïtes Educació nos encontramos en un proceso de transformación educativa denominado Horizonte+. Una evolución lógica del Horizonte 2020, el cual integra los aprendizajes y la experiencia adquirida durante estos años.

Horitzonte+ es un proyecto de innovación para la mejora que busca la profundidad y sitúa al alumnado en el centro del proceso de aprendizaje, poniendo el foco en su desarrollo integral para que sea capaz de conducir la propia vida con sentido y desde la libertad. La educación en ciudadanía global - para poder comprender qué pasa en el mundo y cultivar una mirada crítica que impulse la transformación del entorno con criterios de justicia y equidad - es uno de los principales motores de la innovación que promueve el modelo educativo de Jesuites Educació.

Para nosotros y nosotras, la innovación se ubica dentro de un proceso de transformación que nos conduce a desarrollar nuestro proyecto educativo y mejorar los procesos de enseñanza y aprendizaje, así como la propia cultura institucional. Preguntarnos y redefinir la escuela que queríamos fue un elemento crucial que guió y guía cualquier paso de nuestra acción educativa. Y todo ello pasa, ineludiblemente, por reconocer y reforzar el papel central del educador en este proceso.

\section{Rol y competencias docentes}

En la era del conocimiento y la información, en la era de internet y la era digital, el docente y los equipos directivos ven modificadas en buena parte sus funciones y responsabilidades, y los centros educativos deben repensar sus metas, su organización y sus metodologías. Los y las docentes se convierten en profesionales que orientan y acompañan, enseñan a pensar, a discernir y valorar la información relevante, a analizar problemas y proveer de contexto, a guiar la autorregulación de su alumnado, a cultivar habilidades, actitudes y valores.

La relevancia de la tarea del educador en el modelo educativo de Jesuites Educació resulta central ante el reto de acompañar el crecimiento integral de la persona para que acontezca la mejor versión de sí misma. Para favorecer el crecimiento y la constitución de nuestro alumnado como ciudadanos globales, capaces y con deseo de transformar el mundo, será necesario, como principio indispensable, que aquellos que los acompañen y los guíen también lo sean.

¿Pueden los docentes promover la ciudadanía global si ellos mismos en su esfera pública y privada no la ejercen? ¿Pueden los centros educativos marcar como reto la transformación social de sus niños y jóvenes si no cuentan con estructuras organizativas participativas que lo favorezcan? 
La aparición de este concepto de ciudadanía global no es solo para nuestro alumnado sino para cualquier habitante del planeta. Esto nos lleva a considerar que también debemos acompañar a nuestros educadores y educadoras en desarrollar su dimensión de ciudadano global, de tal manera que su identidad como persona se actualice o modifique. Es por tanto un gran reto el que se nos presenta: formar a nuestros docentes en ciudadanía global a nivel profesional pero también personal, en una doble dimensión que evidentemente se retroalimenta.

Sin lugar a dudas, las nuevas funciones del rol docente requieren que los educadores y educadoras continúen reafirmando, desarrollando y actualizando todo un conjunto de competencias, habilidades y actitudes prioritarias para poder desarrollar su tarea profesional en plenitud. Evidentemente, estas competencias, entre otras, deberán incluir aquellas mismas que deseamos desarrollar en nuestro alumnado.

A continuación, destacamos algunas competencias que consideramos clave en el desarrollo de procesos de innovación:

\section{Competencias docentes claves en procesos de transformación educativa}

1 Compromiso con la educación, la comunidad y el proyecto de escuela.

- Desarrollo de su dimensión de ciudadanía global.

1 Actitud abierta para la cultura de la transformación.

- Capacidad reflexiva y análisis crítico de la propia práctica docente.

1 Trabajo en equipo y cultura colaborativa.

1 Corresponsabilidad y proactividad en la busca de soluciones creativas.

1 Liderazgo para gestionar sus propios desafíos e influir favorablemente en el resto.

- Gestionar la progresión de los aprendizajes de los alumnos y diseñar itinerarios personalizados.

Fuente: propia

\section{Formación docente en procesos de innovación educativa}

La cultura de transformación que rige nuestras escuelas requiere procesos que contribuyan a modelar el aprendizaje profesional de nuestros educadores y
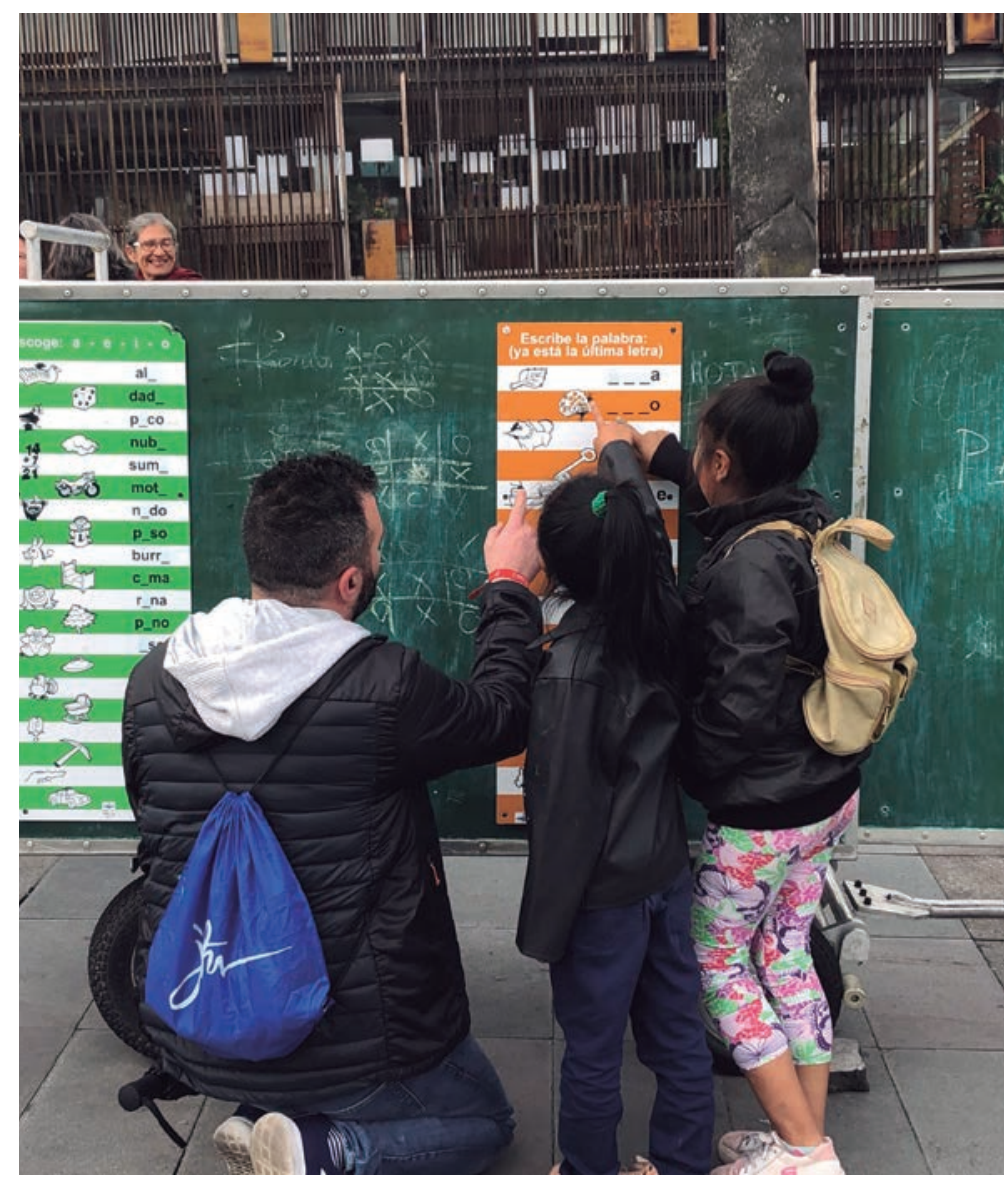

educadoras para una actualización, experimentación y autoevaluación constante de su práctica profesional. En la misma dirección, organismos internacionales como la UNESCO, la OCDE y la OEI también apuntan a la necesidad de encontrar maneras de mejorar el acompañamiento profesional docente para dar una mejor respuesta a los retos que la educación plantea. Congruentemente, la evidencia empírica ha demostrado que la calidad del educador es un factor vital para lograr mejoras en los resultados escolares.

Desde este contexto, se hace necesario una nueva mirada a las experiencias de aprendizaje que ofrecemos a nuestros educadores y educadoras para desarrollar las competencias claves descritas en el anterior apartado y lograr, desde la excelencia sostenible, los retos de nuestro proyecto en constante evolución.

Tal como sugieren varias investigaciones, los programas de acompañamiento con un mayor impacto a largo plazo son aquellos que inciden en la identidad profesional del docente. Será conveniente que las experiencias de desarrollo profesional que ofrecemos a nuestros docentes va-
Participante en el curso de Innovación para la transformación social durante el encuentro en Ecuador 
yan dirigidas a incidir en la identidad profesional que caracteriza a un docente en relación con su rol, sus concepciones, sus procedimientos y estrategias y sus sentimientos de auto-eficacia y auto-concepto:

\section{Elementos que configuran la identidad docente}

1 El rol profesional: referido a las funciones que el docente considera que debe ejercer. Tiene un fuerte componente institucional.

I La práctica de enseñanza y aprendizaje: creencias y concepciones que posee el docente sobre qué significa enseñar y aprender.

1 Y los sentimientos asociados a la docencia: procesos afectivos que impulsan y provocan la actuación docente, tanto de signo positivo (motivación) como negativo (inseguridad, vulnerabilidad), y que están vinculados al reconocimiento profesional, a la autoevaluación personal y a la evaluación institucional.

Fuente: Monereo, C., Weise, C., Álvarez, I. (2013). Cambiar la identidad docente en la universidad. Formación basada en incidentes dramatizados. Infancia y Aprendizaje: Journal for the Study of Education and Development, 36(3), 323-340.

\section{Características y orientaciones de la formación continua}

La formación docente, inicial y particularmente en servicio, solo resultará transformadora de prácticas pedagógicas si es que se realiza lo más cerca posible del aula.

(Elmore, 2010, p. 8)

En coherencia con el paradigma pedagógico ignaciano y el modelo educativo que guía la transformación de nuestras escuelas, los equipos docentes deben tomar un papel activo en las experiencias de aprendizaje en las que participen. Desde este enfoque, se convierte en un requisito partir de la experiencia personal y profesional de los docentes y es necesario que se realice en interacción con otros iguales, reforzando el carácter esencialmente social del aprendizaje.

La reflexión en y sobre la práctica se quiere convertir en un hábito consciente de nuestros educadores, de tal manera que se integre en su actividad diaria y que los conduzca a una visión profunda y crítica de la propia práctica. A la vez que se es- tablecen puentes de unión entre la realidad cotidiana del docente, las disciplinas teóricas y las evidencias aportadas por la investigación reciente.

La formación para un educador hoy debería construirse y articularse a partir del propósito de la institución educativa, de cuáles son los aprendizajes que debe desarrollar o adquirir el alumnado y basándonos en las evidencias y conocimientos de cómo se aprende.

A continuación, recogemos algunas orientaciones a tener presentes en la formación continua a docentes que implementan la innovación en sus aulas:

\section{Recomendaciones y claves en la formación continua}

1 Vincular la experiencia formativa al propósito de la educación y a la mejora de los aprendizajes de nuestro alumnado.

1 Personalizar la experiencia formativa atendiendo a los intereses, necesidades y objetivos de los docentes.

1 Partir del escenario profesional y experiencia personal de los participantes.

1 Contemplar evidencias externas o internas que permitan su contraste y análisis.

- Promover la reflexión sobre la acción en un entorno de aprendizaje colaborativo.

1 Promover un papel activo de los docentes.

- Documentar los procesos seguidos para el análisis del mismo docente y el centro, como para mostrar, difundir y compartir en red.

I Proponer experiencias con continuidad y seguimiento a través de un plan de acción y evaluación.

Fuente: propia

\section{Escenarios formativos}

Podemos identificar diversidad de formatos y experiencias formativas, combinables entre ellas, que nos pueden ayudar a alcanzar el objetivo de consolidar la innovación en nuestro centro educativo:

त Comunidades profesionales de aprendizaje: en los centros educativos, organizados como comunidad de aprendizaje, todas sus instancias y miembros están comprometidos con la construcción y adquisición de nuevos conocimientos y habilidades. 


\section{IIII. ÁQORA DE PROFESORES}

Están conformadas por un grupo de personas que profundizan su conocimiento y experiencia a través de una interacción continua que fortalece sus relaciones; crea conocimiento compartido y potencia el desarrollo profesional. Estas comunidades ayudan a la implementación de innovaciones y también a su sostenibilidad en los centros gracias a la estructura de interacción colaborativa entre todos sus componentes.

入 Autoformación virtual: acceso a plataformas con módulos graduales que incluyen diferentes recursos (videos, documentos, presentaciones...) y promueven la interacción a través de foros tutorizados. Permiten el acceso desde diferentes lugares y en tiempos diferidos.

त Formación presencial instruida por un profesional experto en el tema que usa modalidades variadas de aprendizaje y enseñanza. Tiene capacidad para observar, modelar y evaluar la actuación docente en el aula con el alumnado.

入 Mentoría: relación de desarrollo personal y profesional en la cual un docente novel en ese ámbito recibe el acompañamiento de un profesional con mayor conocimiento y experiencia.

\section{Una experiencia práctica: curso de Innovación para la transformación social}

Durante los cursos académicos 2017 2019, participamos en una experiencia formativa en la red internacional educativa de la Compañía de Jesús con el sugerente nombre de Innovación para la transformación social. En ella participaron un equipo de diferentes docentes y directivos de Latinoamérica (Colombia, Ecuador, Guatemala) y España (Barcelona, Madrid, Palma de Mallorca, Sevilla y Úbeda) coordinado por un equipo multidisciplinar pertenecientes a EDUCSI, Fe y Alegría, Entreculturas y Alboan.

El curso, de un total de 200 horas, tenía parte presencial y parte online proporcio-
Condiciones para favorecer la consolidación de las comunidades profesionales de aprendizaje:

$\searrow$ Visión y valores compartidos.

$\searrow$ Responsabilidad colectiva.

$\searrow$ Confianza mutua, respeto y apoyo.

\ Inclusión de todos los miembros.

$\searrow$ Colaboración centrada en el aprendizaje.

\ Condiciones para la colaboración.

$\searrow$ Liderazgo compartido y enfocado al apoyo.

$\searrow$ Aprendizaje intencional tanto colectivo como individual.

$\searrow$ Reflexión e investigación sobre la propia práctica.

$\checkmark$ Apertura, red y asociaciones.

nando un entorno idóneo para compartir y reflexionar prácticas sobre la educación que deseamos para el mundo actual. Su objetivo principal fue impulsar el conocimiento y la práctica en red de los docentes y directivos participantes orientando su acción hacia una innovación educativa que esté al servicio de la transformación social. Específicamente, se establecieron tres focos de acción:

入 Formar personas comprometidas con la transformación social para mejorar realidades nacionales y globales.

7 Enriquecerse del trabajo en red y fomentar encuentros improbables entre escuelas.

入 Promover el aprendizaje de contenidos vinculados con la innovación y trabajados desde la propia experiencia.

Los dos encuentros presenciales, uno en España y el otro en Ecuador, permitieron el conocimiento del contexto de los distintos asistentes al curso y la generación de lazos y vínculos entre los participantes que abrieron la puerta a la colaboración entre centros. La diversidad de contextos en los que se encuentran los colegios en Latinoamérica y España representó una plataforma inmejorable para la promoción de unos encuentros improbables y el aprendizaje colaborativo de prácticas innovadoras desde diferentes lugares del mundo pero con un mismo propósito: contribuir a la transformación social de nuestro contexto. 
